\title{
CAPACIDAD ANTAGÓNICA DE Trichoderma spp. NATIVA CONTRA Phytophthora parasitica Y Fusarium oxysporum AISLADOS DE CULTIVOS DE JAMAICA
}

\author{
ANTAGONISTIC CAPACITY OF NATIVE Trichoderma spp. AGAINST Phytophthora \\ parasitica AND Fusarium oxysporum ISOLATED FROM ROSELLE CROPS
}

\author{
Alejandro C. Michel-Aceves ${ }^{1}$, Javier Hernández-Morales ${ }^{2}$, Rocío Toledo-Aguilar ${ }^{3}$, \\ Juan E. Sabino López ${ }^{4}$ y Teolincacihuatl Romero-Rosales ${ }^{4}$ *
}

\begin{abstract}
'Colegio Superior Agropecuario del Estado de Guerrero, Cocula, Guerrero, México. ${ }^{2}$ Colegio de Postgraduados, Instituto de Fitosanidad, Montecillo, Texcoco, Estado de México, México. ${ }^{3}$ Instituto Nacional de Investigaciones Forestales, Agrícolas y Pecuarias, Campo Experimental Iguala, Iguala, Guerrero, México. ${ }^{4}$ Universidad Autónoma de Guerrero, Unidad Académica de Ciencias Agropecuarias y Ambientales, Iguala, Guerrero, México.
\end{abstract}

*Autor de correspondencia (romerot@colpos.mx)

\section{RESUMEN}

La enfermedad Pata Prieta en jamaica (Hibiscus sabdariffa L.) es ocasionada principalmente por Phytophthora parasitica Dastur y Fusarium oxysporum. Estos patógenos causan pérdidas de producción en al menos $50 \%$ en el estado de Guerrero, donde se siembra la mayor superficie de este cultivo en México. Los fungicidas que se emplean para el control de la enfermedad son caros, tóxicos y residuales, por lo que es necesario buscar alternativas de control dentro de un contexto de agricultura sustentable. El objetivo de este estudio fue evaluar la capacidad antagónica in vitro de cepas nativas de Trichoderma spp. contra $P$. parasitica y F. oxysporum. Las confrontaciones duales entre patógenos y agentes antagónicos se evaluaron en cajas Petri bajo un diseño experimental completamente al azar con cuatro repeticiones. Las variables registradas fueron días al primer contacto entre hifas, zona de intersección, tipo de antagonismo y porcentaje de inhibición. La información fue sometida a análisis de varianza y prueba de comparación de medias (Tukey, $\mathrm{P} \leq 0.05$ ). Las cinco cepas de Trichoderma sobrecrecieron a $P$. parasitica y cubrieron $100 \%$ del medio; en el caso de la confrontación con $F$. oxysporum, Trichoderma creció sólo en dos terceras partes del medio. En las zonas de intersección se observó variación de 0.0 a $4.8 \mathrm{~cm}$ entre patógenos y antagónicos. La confrontación entre $T$. inhamatum cepa 14 y $P$. parasitica mostró el mayor contacto entre hifas con $4.82 \mathrm{~cm}$; Ti14, Ta10 y Ta9 mostraron el primer contacto entre hifas a los 3 y $4 \mathrm{~d}$ en $P$. parasitica y $F$. oxysporum; la primera cepa tuvo un porcentaje de inhibición superior con 95 y $85 \%$ para los dos patógenos. Las cepas Ti14 y Ta10 mostraron mayor antagonismo contra ambos patógenos por el tiempo al primer contacto, esporulación, crecimiento sobre los patógenos y porcentaje de inhibición. Las cepas nativas de Trichoderma representan una alternativa viable para el control de la pata prieta en Jamaica.

Palabras clave: Hibiscus sabdariffa, antibiosis, control biológico, micoparasitismo, Pata Prieta.

\section{SUMMARY}

The black leg disease in roselle (Hibiscus sabdariffa L.) is mainly caused by Phytophthora parasitica Dastur and Fusarium oxysporum Schltdl. These pathogens cause losses in production of at least $50 \%$ in the state of Guerrero, where the largest area of this crop is planted in Mexico. Fungicides used to control this disease are expensive, toxic and residual; thus, it is necessary to look for control alternatives within a context of sustainable agriculture. The objective of this study was to evaluate the in vitro antagonistic capacity of native strains of Trichoderma spp. against $P$. parasitica and F. oxysporum. Dual confrontations between pathogens and antagonistic agents were evaluated in Petri dishes under a completely randomized experimental design with four replications. The traits recorded were days at the first contact between hyphae, intersection zone, type of antagonism and percentage of inhibition The information was submitted to analysis of variance and comparison test of means (Tukey, $\mathrm{P} \leq 0.05$ ). The five strains of Trichoderma overgrew $P$. parasitica and covered $100 \%$ of the medium; in the case of the confrontation with $F$. oxysporum, Trichoderma grew only in two thirds of the medium. In the intersection zones, a variation of 0.0 to $4.8 \mathrm{~cm}$ was observed between pathogens and antagonists. The confrontation between $T$. inhamatum strain 14 and $P$. parasitica showed the largest contact between hyphae with 4.82 $\mathrm{cm}$; Ti14, Ta10 and Ta9 showed the first contact between hyphae at 3 and $4 \mathrm{~d}$ in $P$. parasitica and F. oxysporum; the first strain had a higher inhibition percentage with 95 and $85 \%$ for both pathogens. The Ti14 and Ta10 strains showed greater antagonism against both pathogens by time at first contact, sporulation, growth on the pathogens and percentage of inhibition Trichoderma native strains represent a viable alternative for black leg control in roselle.

Index words: Hibiscus sabdariffa, antibiosis, biological control, mycoparasitism, black leg.

\section{INTRODUCCIÓN}

En el estado de Guerrero, México la enfermedad Pata Prieta en jamaica (Hibiscus sabdariffa L.), ocasionada por Phytophthora parasitica Dastur y Fusarium oxysporum Schltdl, es una de las principales limitantes fitosanitarias del cultivo, con pérdidas de producción superiores a 50 \% (Hassan et al., 2014; Ortega-Acosta et al., 2015) Los métodos de control químico, para esta y otras enfermedades, han demostrado ser eficientes, pero sus efectos secundarios son cuestionados por su impacto negativo al ambiente y a la salud humana y por la resistencia que desarrollan los patógenos a los ingredientes activos (Veena y Reddy, 2014; Jeyaseelan et al., 2012).

El biocontrol de enfermedades tiene como propósito cambiar el equilibrio a favor del ecosistema, mediante un incremento artificial de microorganismos benéficos 
contra poblaciones patógenas con la finalidad de favorecer la producción agrícola (Harman, 2006). El género Trichoderma comprende varias especies de hongos filamentosos que han sido usados como agentes de biocontrol en enfermedades de importancia para la agricultura y constituyen una alternativa al control químico (Bhale et al., 2013; Ghazanfar et al., 2018); estos hongos, con uso potencial para la agricultura, se pueden encontrar en el suelo, madera en descomposición y material vegetal y existe amplia diversidad dentro y entre especies (Kredics et al., 2014).

La actividad antagónica de las especies de Trichoderma involucran mecanismos de acción como microparasitismo, antibiosis, competencia por nutrientes, entre otros (Ghazanfar et al., 2018; Mungole y Chaturvedi, 2011); además, las especies de Trichoderma muestran acción biorreguladora de forma indirecta, promueven mecanismos de defensa fisiológicos y bioquímicos en la planta para inducir resistencia sistémica a enfermedades, mejoran el crecimiento y desarrollo de plantas (Ghazanfar et al., 2018; Guzmán-Guzmán et al., 2019), incrementan la tolerancia de la planta a estrés por sequía o salinidad y solubilizan elementos nutritivos que en su forma original no son accesibles para las plantas (Kashyap et al., 2017; López-Bucio et al., 2015).

La diversificación de estos mecanismos permite que las especies de Trichoderma sean colonizadores exitosos en sus hábitats, lo cual se refleja en el uso eficiente de sustratos y en la liberación de metabolitos secundarios y enzimas (Schuster y Schmoll, 2010). En este contexto, el objetivo de la presente investigación fue evaluar la capacidad antagónica in vitro de cepas nativas de Trichoderma spp. aisladas de suelo con cultivo de jamaica en la Costa Chica de Guerrero, contra P. parasitica y F. oxysporum, agentes causales de la enfermedad Pata Prieta en jamaica.

\section{MATERIALES Y MÉTODOS}

\section{Cepas estudiadas}

Se utilizaron cinco cepas nativas antagónicas, cuatro de Trichoderma asperellum (Ta6, Ta9, Ta10 y Ta11) y una de T. inhamatum (Ti14), aisladas de suelo cultivado con jamaica de los municipios de Tecoanapa y Ayutla, Guerrero, identificadas morfológica y taxonómicamente (Gams y Bisett, 1998).

Las cepas de los hongos fitopatógenos P. parasitica y F. oxysporum fueron proporcionadas por el Colegio de Postgraduados, Campus Montecillo, aisladas y caracterizadas morfológica y molecularmente, con los números de depósito KM519189 y KM519188 en el
NCBI (Ortega-Acosta et al., 2015); estas cepas fueron cultivadas en el laboratorio de Biotecnología del Colegio Superior Agropecuario del estado de Guerrero, en papadextrosa-agar (PDA, Sigma®) y medio de cultivo con jugo de verduras $\mathrm{V} 8{ }^{\circledR}$. La incubación se realizó a temperatura ambiente en cultivo estático con 12 h luz/oscuridad, durante $10 \mathrm{~d}$; transcurrido este tiempo, se realizaron los ensayos de antagonismo.

\section{Actividad antagónica in vitro de cepas de Trichoderma}

Los cultivos duales para evaluar la actividad antagónica de los hongos nativos de Trichoderma contra los patógenos se realizaron in vitro, con 10 interacciones en total y sus testigos sin confrontación. Se usó un diseño experimental completamente al azar con cuatro repeticiones; la unidad experimental fue una caja Petri, donde se desarrollaron las confrontaciones.

En cada caja Petri con medio de cultivo PDA se depositó un disco de $5 \mathrm{~mm}$ de diámetro con micelio de los hongos patógenos (P. parasitica y F. oxysporum) y en crecimiento activo; cada disco se ubicó a $2 \mathrm{~cm}$ del extremo de la caja. Dos días después, en forma equidistante, se colocó un disco de micelio de $5 \mathrm{~mm}$ de diámetro de cada aislado de Trichoderma spp. con 10 d de crecimiento. La incubación se realizó a temperatura ambiente $\left(\approx 23^{\circ} \mathrm{C}\right)$ con $12 \mathrm{~h}$ luz/ oscuridad y se observaron cada $24 \mathrm{~h}$ para registrar las variables siguientes: días al primer contacto entre hifas, zona de intersección, tipo de antagonismo, capacidad de esporulación de las especies de Trichoderma sobre las cepas de P. parasitica y F. oxysporum e interacción del organismo antagónico con el patógeno.

Cada tratamiento se clasificó con la escala 1 a 5 de antagonismo descrita por Bell et al. (1982); donde 1) Trichoderma spp. sobrecrece completamente al patógeno y cubre totalmente la superficie del medio, 2) Trichoderma spp. sobrecrece dos terceras partes de la superficie del medio, 3) Trichoderma spp. y el patógeno colonizan cada uno aproximadamente la mitad de la superficie y ningún organismo domina al otro, 4) el patógeno coloniza dos terceras partes de la superficie del medio y parece resistir la invasión por Trichoderma spp. y 5) el patógeno sobrecrece completamente a Trichoderma spp. y cubre totalmente la superficie del medio.

La zona de intersección, descrita como el crecimiento del antagónico sobre el patógeno, se registró después del primer contacto entre hifas. Al observar crecimiento micelial, se realizaron mediciones con una regla en $\mathrm{cm}$. Se midió la zona de traslape o intersección de las colonias, desde el disco de agar de Trichoderma spp. hasta la punta 
final del micelio; las mediciones se registraron cada $24 \mathrm{~h}$, hasta que el testigo cubrió la superficie de la caja Petri.

La competencia por nutrientes y espacio se valoró al comparar la velocidad del crecimiento; se midió el radio de crecimiento de patógenos (RCP) y antagonistas (RCA) con la ayuda de un calibrador Pie de Rey (Stefanova, 2007). Al mismo tiempo, se evaluó el porcentaje de inhibición de crecimiento radial $(P I C R)$ con la fórmula $\mathrm{PICR}=(\mathrm{R} 1-\mathrm{R} 2) /$ $R 1 \times 100$, donde $R 1$ es el radio del patógeno testigo y $R 2$ es el radio del patógeno en enfrentamiento (Ezziyyani et al., 2004). Los datos se sometieron a análisis de varianza y prueba de comparación de medias múltiple (Tukey, $\mathrm{P} \leq$ 0.05) con el paquete estadístico SAS V9.3 (SAS Institute, 2011).

\section{RESULTADOS Y DISCUSIÓN}

\section{Contacto entre hifas}

En los tratamientos confrontados, las hifas de ambos microorganismos hicieron contacto en promedio a los $3 \mathrm{y}$ 4 días después de la siembra; se observó nulo crecimiento al momento del contacto con el agente antagónico en los aislados de P. parasitica y F. oxysporum. Este efecto se reportó contra Alternaria porri, al confrontarse con T. harzianum en pruebas in vitro, donde el fitopatógeno detuvo su crecimiento al contacto con el antagonista, que también mostró fuerte actividad micoparasítica y alta capacidad competitiva por espacio y nutrientes (AboElyousr et al., 2014). Al respecto, Vargas-Hoyos y GilchristRamelli (2015) mencionaron que cepas de T. asperellum mostraron habilidad para adaptarse a condiciones de crecimiento in vitro y colonizar en poco tiempo a Fusarium sp., lo que causó la inhibición del crecimiento del patógeno.

\section{Zona de intersección}

En las Figuras 1 y 2 se muestra el crecimiento dual de las especies de Trichoderma spp. sobre F. oxysporum y $P$. parasítica, respectivamnte. En las zonas de intersección se registraron variaciones de distancia entre 0.0 y $4.8 \mathrm{~cm}$. La confrontación de T. inhamatum cepa 14 contra P. parasitica, (Ti14P) mostró la mayor intersección con $4.82 \mathrm{~cm}$, seguido por Ta10P (T. asperellum cepa 10 contra P. parasitica) con $4.77 \mathrm{~cm}$. Con F. oxysporum, estas dos cepas antagónicas de Trichoderma también presentaron los mejores resultados con una intersección de 4.11 y 3.46 cm (Cuadros 1 y 2). Cuando Trichoderma tiene el primer contacto con el hongo patógeno activa sus mecanismos de acción para colonizar al hospedero, y en algunos casos, como en el presente estudio, las cinco cepas de Trichoderma sobrecrecieron y redujeron a $P$. parasitica, mostrando evidencia de su alta capacidad micoparasítica por el enrollamiento y formación de apresorios y producción de quitinasas y glucanasas (Ghazanfar et al., 2018).
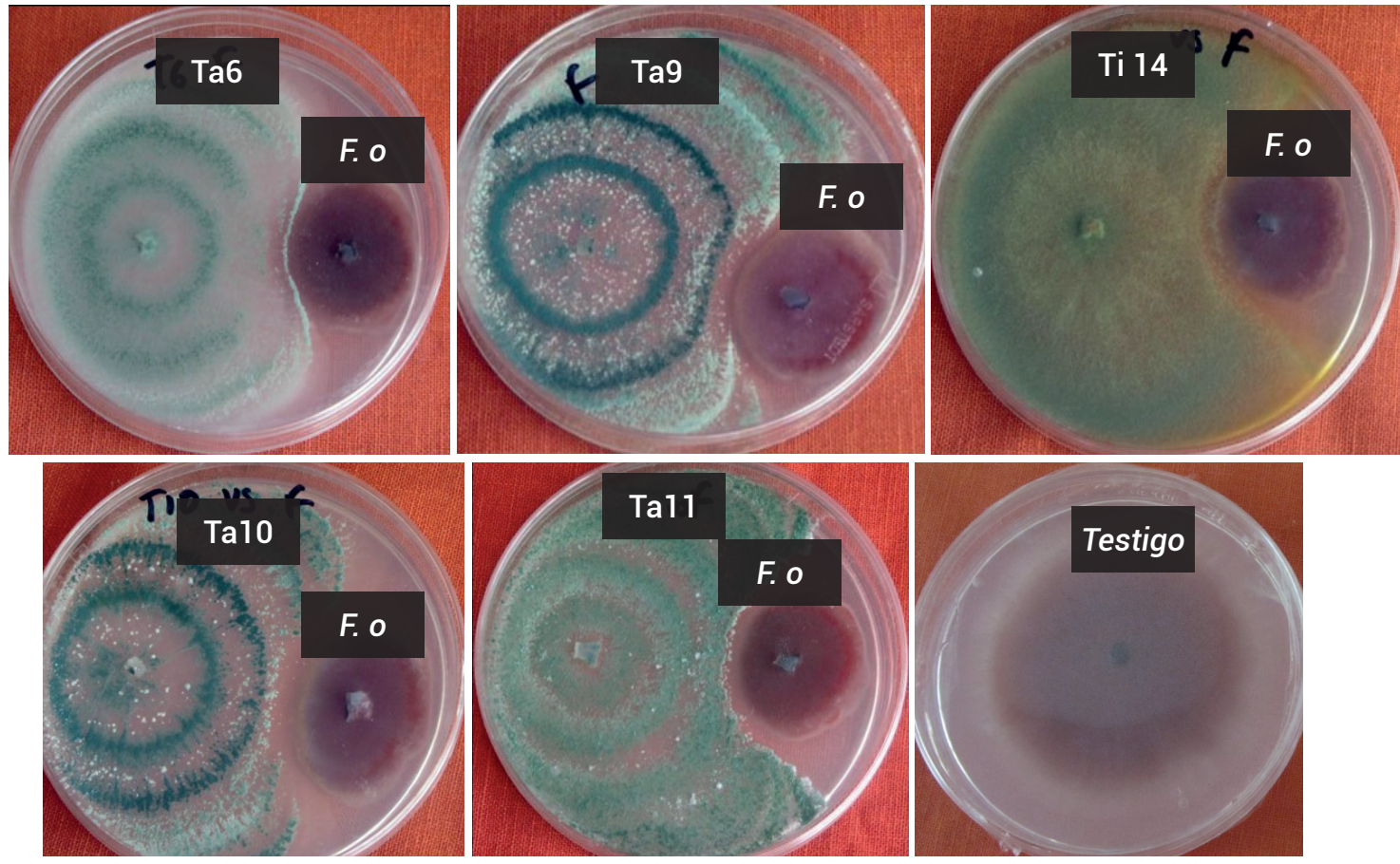

Figura 1. Cultivos duales de Trichoderma asperellum (Ta6, Ta9, Ta10 y Ta11) y T. inhamatum (Ti14) contra Fusarium oxysporum (F.o). 

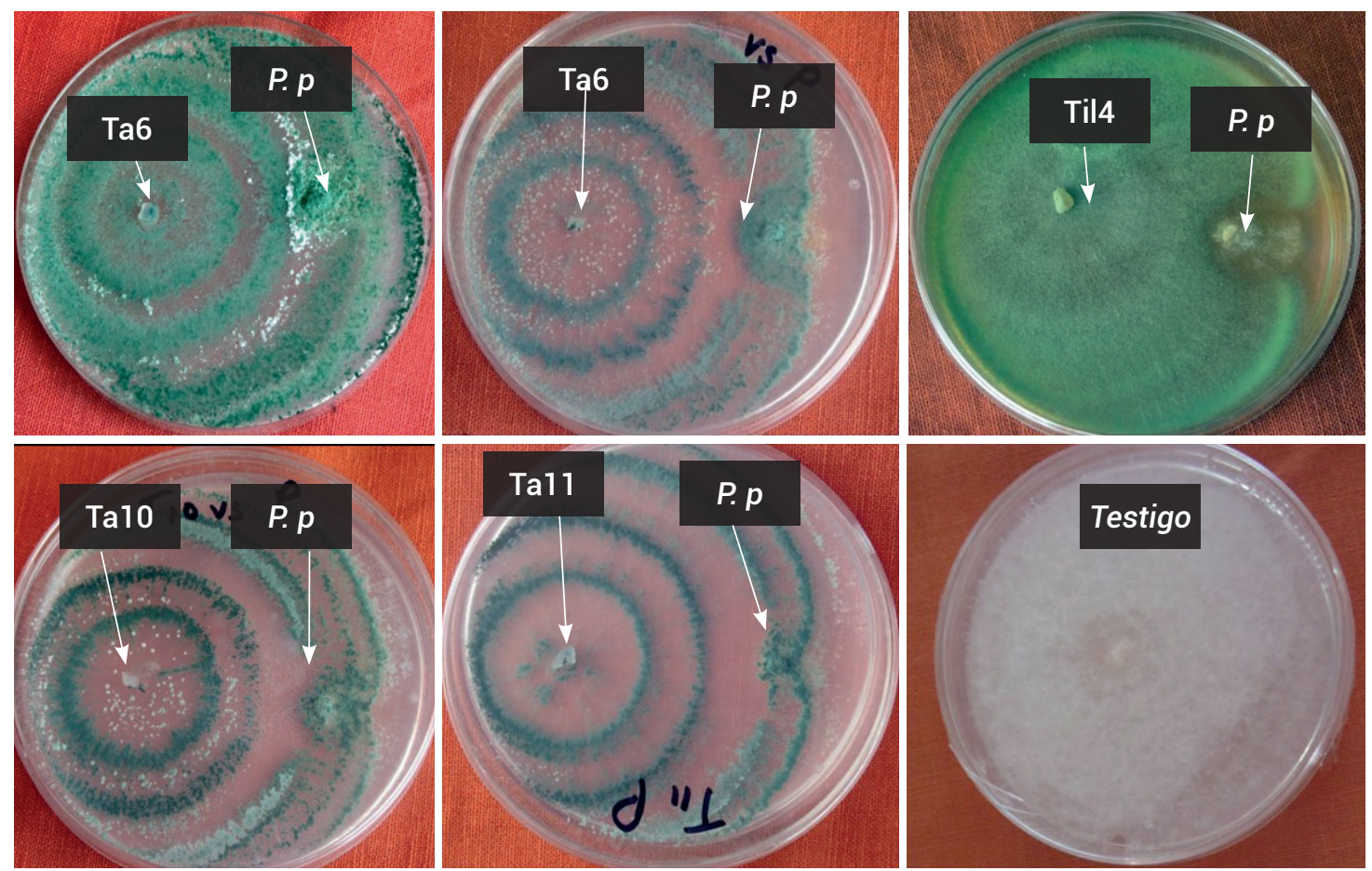

Figura 2. Cultivos duales de Trichoderma asperellum (Ta6, Ta9, Ta10 y Ta11) y T. inhamatum (Ti14) contra Phythopthora parasitica (P. p).

Cuadro 1. Contacto, sobrecrecimiento y esporulación de especies de Trichoderma sobre Fusarium oxysporum.

\begin{tabular}{|c|c|c|c|c|c|c|c|}
\hline \multirow[b]{2}{*}{ Cepa } & \multirow[b]{2}{*}{ Especie } & \multirow[b]{2}{*}{$\mathrm{DPC}^{+}$} & \multirow[b]{2}{*}{ Inhibición } & \multicolumn{2}{|c|}{ Esporulación } & \multirow{2}{*}{$\begin{array}{c}\text { Clase de } \\
\text { antagonismo }\end{array}$} & \multirow[b]{2}{*}{${ }^{\circ} \mathrm{CSP}(\mathrm{cm}$} \\
\hline & & & & Trichoderma spp. & $\begin{array}{l}\text { Fusarium } \\
\text { oxysporum }\end{array}$ & & \\
\hline Ti14 & T. inhamatum & $4 a$ & + & + & - & 2 & $4.11 \mathrm{a}$ \\
\hline Ta10 & T. asperellum & $4 a$ & + & + & - & 2 & $3.46 b$ \\
\hline Ta9 & T. asperellum & $4 a$ & + & + & - & 2 & $3.22 b$ \\
\hline Ta6 & T. asperellum & $5 b$ & + & + & - & 2 & $3.19 b$ \\
\hline Ta11 & T. asperellum & $5 b$ & + & + & - & 2 & $3.11 \mathrm{C}$ \\
\hline
\end{tabular}

${ }^{\dagger}$ DPC: días al primer contacto. ${ }^{+\dagger}$ Clase de antagonismo: 2 (Trichoderma sobrecrece dos terceras partes de la superficie del medio; Bell et al., 1982). "CSP. crecimiento sobre el patógeno. Medias con letras iguales en columnas no son estadísticamente diferentes (Tukey, P $\leq 0.05)$.

Cuadro 2. Contacto, sobrecrecimiento y esporulación de especies de Trichoderma sobre Phytophthora parasitica.

\begin{tabular}{|c|c|c|c|c|c|c|c|}
\hline \multirow[b]{2}{*}{ Cepa } & \multirow[b]{2}{*}{ Especie } & \multirow[b]{2}{*}{$\mathrm{DPC}^{+}$} & \multirow[b]{2}{*}{ Inhibición } & \multicolumn{2}{|c|}{ Esporulación } & \multirow{2}{*}{$\begin{array}{c}\text { Clase de } \\
\text { antagonismo }\end{array}$} & \multirow[b]{2}{*}{${ }^{\circ} \mathrm{CSP}(\mathrm{cm})$} \\
\hline & & & & Trichoderma spp. & $\begin{array}{c}\text { Phytophthora } \\
\text { parasitica }\end{array}$ & & \\
\hline Ti14 & T. inhamatum & $3 a$ & + & + & - & 1 & $4.82 \mathrm{a}$ \\
\hline Ta10 & T. asperellum & $3 a$ & + & + & - & 1 & $4.77 b$ \\
\hline Ta9 & T. asperellum & $3 a$ & + & + & - & 1 & $4.65 \mathrm{c}$ \\
\hline Ta6 & T. asperellum & $4 b$ & + & + & - & 1 & $4.48 d$ \\
\hline Ta11 & T. asperellum & $4 b$ & + & + & - & 1 & $4.48 \mathrm{~d}$ \\
\hline
\end{tabular}

${ }^{\dagger} \mathrm{DPC}$ : días al primer contacto. ${ }^{\dagger+}$ Clase de antagonismo: 1 (Trichoderma sobrecrece completamente al patógeno y cubre totalmente la superficie del medio; Bell et al., 1982). "CSP. crecimiento sobre el patógeno. Medias con letras iguales en columnas no son estadísticamente diferentes (Tukey, P $\leq 0.05)$. 
En los cultivos duales donde se presentaron zonas de interacción entre antagonistas y los dos patógenos se observó que entre mayor fue la intersección se desarrolló mayor hiperparasitismo de los aislados antagónicos sobre P. parasitica. En un estudio con T. harzianum vs. P. capsici la zona de inhibición aumentó a medida que transcurrió el tiempo por la destrucción del micelio del patógeno y algunas interacciones mostraron una zona de inhibición progresiva por la velocidad de crecimiento del antagonista (Ezziyyani et al., 2004). La reducción en la tasa de crecimiento del patógeno en cultivos duales es un indicador de la capacidad antagónica de Trichoderma y puede visualizarse un aumento en la zona de intersección durante el periodo de incubación y reducción del diámetro de las colonias del patógeno (Guigón-López et al., 2010).

Las diferencias entre las zonas de intersección se deben a la producción de antibióticos volátiles y no volátiles, mismos que se sintetizan al momento del contacto entre antagonista y fitopatógeno (González et al., 2012; Osorio et al., 2016) y juegan un papel importante en el micoparasitismo (Harman, 2006).

\section{Capacidad de esporulación de Trichoderma spp.}

Las cinco cepas de Trichoderma spp. presentaron esporulación, en particular Ta9 frente a P. parasitica y Ta10 frente a F. oxysporum, las cuales esporularon al tercer día después de la siembra en los cultivos duales (Cuadros 1 y 2). En otros ensayos, en pruebas de laboratorio, el comportamiento de aislados de Trichoderma spp. sobre P. capsici retardó el crecimiento al colonizar y esporular sobre el patógeno; es decir, la esporulación fue gradual, pero la colonización de Trichoderma spp. fue rápida por el proceso micoparasítico (Ezziyyani et al., 2005). Esta característica también fue detectada en $T$. harzianum, $T$. koningiopsis y $T$. asperellum contra P. capsici, al reducir la producción de esporangios y el crecimiento micelar de las dos cepas fitopatógenas (Ramírez-Delgado et al., 2018). La inhibición y reducción de la esporulación del patógeno y la capacidad de esporular de Trichoderma es una muestra de su capacidad antagónica para frenar y colonizar a hospederos.

\section{Clasificación del antagonismo}

Las cinco cepas de Trichoderma spp. cubrieron por completo la colonia de P. parasitica y se ubicaron en la Clase 1 de antagonismo. En la confrontación dual entre las cepas de Trichoderma spp. y F. oxysporum todas presentaron la clase de antagonismo 2, donde Trichoderma creció en dos terceras partes de la superficie del medio (Figuras 1 y 2). En estudios con Sclerotium rolfsii también se reportó antagonismo Clase 1 al confrontarse con T. harzianum (Corrêa et al., 2007); sin embargo, otros aislados de T. harzianum mostraron Clase 5 de antagonismo contra el mismo patógeno; esto sugiere que los aislados de la misma especie de Trichoderma tienen comportamientos distintos frente a un mismo organismo fitopatógeno, lo que se atribuye a la selectividad de Trichoderma spp. para producir mayor o menor cantidad de enzimas que hidrolizan paredes celulares, característica que está estrechamente relacionada con la capacidad micoparasítica, producción de compuestos antibióticos y fungistáticos (Ramos et al., 2008; Hermosa et al., 2012). Este comportamiento diferencial de Trichoderma spp. hacia un mismo patógeno también se asocia a la variabilidad entre aislamientos del fitopatógeno aun de la misma especie. Específicamente, T. asperellum produce compuestos como el trichodermin, trichodermol, paracelsin a-d, trichobrachim y toxina $T-2$, entre otros metabolitos volátiles con actividad antifúngica, a los cuales se atribuyen los resultados observados en esta interacción (Parmar et al., 2015).

\section{Inhibición de P. parasitica y F. oxysporum por aislados de Trichoderma spp.}

El porcentaje de inhibición de los cultivos duales se muestra en el Cuadro 3. T. inhamatum (Ti14) mostró una actividad sobresaliente en las pruebas de antagonismo y obtuvo 95 y $85 \%$ de inhibición de P. parasitica y $F$. oxusporum, respectivamente, seguido de Ta10 con 89 y $85 \%$ de inhibición para ambos patógenos. En pruebas con $T$. virens y $T$. harzianum contra $F$. oxysporum y $F$. subglutinans la inhibición del crecimiento de los patógenos fue superior a $60 \%$, por lo que se consideraron como

Cuadro 3. Antagonismo de Trichoderma spp. contra $P$. parasitica y F. oxysporum en cultivos duales.

\begin{tabular}{lcc}
\hline $\begin{array}{c}\text { Cepa } \\
\text { Trichoderma }\end{array}$ & Cultivo dual & $\begin{array}{c}\text { Inhibición } \\
(\%)\end{array}$ \\
\hline Ti14 & T. inhamatum y P. parasitica & $95 \mathrm{a}$ \\
Ti14 & T. inhamatum y F. oxysporum & $85 \mathrm{a}$ \\
Ta10 & T. asperellum y P. parasitica & $89 \mathrm{a}$ \\
Ta10 & T. asperellum y F. oxysporum & $85 \mathrm{a}$ \\
Ta9 & T. asperellum y P. parasitica & $83 \mathrm{a}$ \\
Ta 9 & T. asperellum y F. oxysporum & $78 \mathrm{~b}$ \\
Ta 6 & T. asperellum y P. parasitica & $85 \mathrm{a}$ \\
Ta 6 & T. asperellum y F. oxysporum & $78 \mathrm{~b}$ \\
Ta 11 & T. asperellum y P. parasitica & $82 \mathrm{~b}$ \\
Ta 11 & T. asperellum y F. oxysporum & $76 \mathrm{c}$
\end{tabular}

Medias con letras iguales en columna no son estadísticamente diferentes (Tukey, $\mathrm{P} \leq 0.05$ ). 
agentes potenciales de control biológico contra hongos patógenos causales de la enfermedad escoba de bruja en mango (Mangifera indica L.) (Michel-Aceves et al., 2008). La producción de compuestos antifúngicos que afecta la pared celular del patógeno causa micoparasitismo, detienen su crecimiento y aumentan su inhibición (González et al., 2011).

Con base en los resultados obtenidos, las cepas de Trichoderma spp. son potenciales biocontroladores de los agentes causales de la enfermedad Pata Prieta en jamaica, específicamente Ti14 y Ta10, ya que cumplen con las características esenciales para considerarse como un agente de control biológico eficaz, tales como alto nivel de competencia por espacio y nutrientes, alto micoparasitismo, menor tiempo de contacto entre hifas, mayor zona de intersección, esporulación sobre los patógenos e inhibición de éstos, fuerte actividad antibiótica y producción de sustancias que inducen fungistasis; además, tienen la ventaja de que son cepas nativas de la región productora de jamaica en Guerrero, lo que coadyuva en su efecto, potencial antagónico y control de los principales hongos patógenos del cultivo, ya que uno de los principales problemas en la efectividad del control biológico de fitopatógenos es que los organismos empleados no están adaptados a las condiciones ambientales locales donde serán aplicados y la producción enzimática se ve afectada por la temperatura y condiciones del ambiente (Parmar et al., 2015).

\section{CONCLUSIONES}

Las cepas Ti14 (T. inhamatum) y Ta10 (T. asperellum) se identificaron como potenciales organismos biocontroladores de los principales agenten causales de la Pata Prieta del cultivo de jamaica en Guerrero. Estas cepas mostraron gran potencial antagónico y agresivo en poco tiempo, con la ventaja de estar adaptadas al ambiente de la principal región de producción de jamaica en México, donde existe alta incidencia de esta enfermedad.

\section{BIBLIOGRAFÍA}

Abo-Elyousr K. A. M., S. I. I. Abdel-Hafez and I. R. Abdel-Rahim (2014) Isolation of Trichoderma and evaluation of their antagonistic potential against Alternaria porri. Journal of Phytopathology 162:567-574, https://doi.org/10.1111/jph.12228

BellD.K.,H.D.WellsandC. R.Markham(1982) Invitroantagonism of Trichoderma species against six fungal plant pathogens. Phytopathology 72:379382, https://doi.org/10.1094/Phyto-72-379

Bhale U. N., P. M. Wagh and J. N. Rajkonda (2013) Antagonistic confrontation of Trichoderma spp against fruit rot pathogens on sapodilla (Manilkara zapota L.). Journal of Yeast and Fungal Research 4:5-11, https://doi.org/10.5897/JYFR12.029

Corrêa S., M. Mello, Z. R. Ávila, L. Minaré B., R. R. Pádua y D. Gomes (2007) Cepas de Trichoderma spp. para el control biológico de Sclerotium rolfsii Sacc. Fitosanidad 11:3-9.
Ezziyyani M., C. Pérez S., A. S. Ahmed, M. E. Requena y M. E. Candela (2004) Trichoderma harzianum como biofungicida para el biocontrol de Phytophthora capsici en plantas de pimiento (Capsicum annuum L.). Anales de Biología 26:35-45.

Ezziyyani M., M. E. Requena y M. E. Candela (2005) Producción de proteínas-PR en la inducción de resistencia a Phytophthora capsici en plantas de pimiento (Capsicum annuum L.) tratadas con Trichoderma harzianum. Anales de Biología 27:143-153.

Gams W. and J. Bissett (1998) Morphology and identification of Trichoderma. In: Trichoderma and Gliocladium. Volume 1 Basic Biology, Taxonomy and Genetics. C. P. Kubicek and G. E. Harman (eds.). Taylor and Francis. London, UK. pp:3-34.

Ghazanfar M. U., M. Raza, W. Raza and M. I. Qamar (2018) Trichoderma as potential biocontrol agent, its exploitation in agriculture: a review. Plant Protection 2:109-135.

González I., D. Infante, B. Martínez, Y. Arias, N. González, I. Miranda y B. Peteira (2012) Inducción de quitinasas y glucanasas en cepas de Trichoderma spp. promisorias como agentes para el control biológico. Biotecnología Aplicada 29:7-11.

González I., D. Infante, B. Peteira, B. Martínez, Y. Arias, N. González e I. Miranda (2011) Caracterización bioquímica de aislamientos de Trichoderma spp. promisorios como agentes de control biológico. II. Expresión de actividad glucanasa. Revista de Protección Vegetal 26:23-29.

Guigón-López C., V. Guerrero-Prieto, F. Vargas-Albores, E. Carvajal-Millán, G. D. Ávila-Quezada, L. Bravo-Luna, M. Ruocco, S. Lanzuise, S. Woo y M. Lorito (2010) Identificación molecular de cepas nativas de Trichoderma spp. su tasa de crecimiento in vitro y antagonismo contra hongos fitopatógenos. Revista Mexicana de Fitopatología 28:87-96.

Guzmán-Guzmán P., M. D. Porras-Troncoso, V. Olmedo-Monfil and A. Herrera-Estrella (2019) Trichoderma species: versatile plant symbionts. Phytopathology 109:6-16, https://doi.org/10.1094/PHYTO-07-18-0218-RVW

Harman G. E. (2006) Overview of mechanisms and uses of Trichoderma spp. Phytopathology 96:190-194 https://doi.org/10.1094/PHYTO-96-0190

Hassan N., M. Shimizu and M. Hyakumachi (2014) Occurrence of root rot and vascular wilt diseases in roselle (Hibiscus sabdariffa L.) in upper Egypt. Mycobiology 42:66-72, https://doi.org/10.5941/MYC0.2014.42.1.66

Hermosa R., A. Viterbo, I. Chet and E. Monte (2012) Plant-beneficial effects of Trichoderma and of its genes. Microbiology 158:17-25, https://doi.org/10.1099/mic.0.052274-0

Jeyaseelan E. C., S. Tharmila and K. Niranjan (2012) Antagonistic activity of Trichoderma spp. and Bacillus spp. against Pythium aphanidermatum isolated from tomato damping off. Archives of Applied Science Research 4:1623-1627.

Kashyap P. L., P. Rai, A. K. Srivastava and S. Kumar (2017) Trichoderma for climate resilient agriculture. World Journal of Microbiology and Biotechnology 33:155, https://doi.org/0.1007/s11274-017-2319-1

Kredics L., L. Hatvani, S. Naeimi, P. Körmöczi, L. Manczinger, C. Vágvölgyi and I. Druzhinina (2014) Biodiversity of the genus Hypocrea/ Trichoderma in different habitats. In: Biotechnology and Biology of Trichoderma. V. K. Gupta, M. Schmoll, A. Herrera-Estrella, R. S. Upadhyay, I. Druzhinina and M. G. Tuohy (eds.). Elsevier. Amsterdam, The Netherlands. pp:324, https://doi.org/10.1016/B978-0-444-59576-8.00001-1

López-Bucio J., R. Pelagio-Flores and A. Herrera-Estrella (2015) Trichoderma as biostimulant: exploiting the multilevel properties of a plant beneficial fungus. Scientia Horticulturae 196:109-123, https://doi.org/10.1016/j.scienta.2015.08.043

Michel-Aceves A. C., M. A. Otero-Sánchez, R. D. Martínez-Rojero, R. ArizaFlores, A. Barrios-Ayala y A. Rebolledo-Martínez (2008) Control biológico in vitro de enfermedades fungosas en tomate Lycopersicum esculentum Mill. Avances en Investigación Agropecuaria 12:55-68.

Mungole A. and A. Chaturvedi (2011) Hibiscus sabdariffa L. a rich source of secondary metabolites. International Journal of Pharmaceutical Sciences Review and Research 6:83-87.

Ortega-Acosta S. A., J. Hernández-Morales, J. S. Sandoval-Islas, V. AyalaEscobar, L. Soto-Rojas y A. Alejo-Jaimes (2015) Distribución y frecuencia de organismos asociados a la enfermedad "Pata 
Prieta" de la jamaica (Hibiscus sabdariffa L.) en Guerrero, México. Revista Mexicana de Fitopatología 33:173-194.

Osorio H. E., F. D. Hernández C., R. Rodríguez H., S. E. Varela F., B. Estrada D. y J. A. López S. (2016) Actividad antagónica de Trichoderma spp. sobre Rhizoctonia solani in vitro. Investigación y Ciencia 24:5-11

Parmar H. J., N. P. Bodar, H. N. Lakhani, S. V. Patel, V. V. Umrania and M. M. Hassan (2015) Production of lytic enzymes by Trichoderma strains during in vitro antagonism with Sclerotium rolfsii, the causal agent of stem rot of groundnut. African Journal of Microbiology Research 9:365-372, https://doi.org/10.5897/AJMR2014.7330

Ramírez-Delgado E., J. J. Luna-Ruíz, 0. Moreno-Rico, J. D. C. QuirozVelásquez and J. L. Hernández-Mendoza (2018) Effect of Trichoderma on growth and sporangia production of Phytophthora capsici. Journal of Agricultural Science 10:815, https://doi.org/10.5539/jas.v10n6p8

Ramos A. S., S. B. Fiaux and S. G. F. Leite (2008) Production of 6-pentyl-apyrone by Trichoderma harzianum in solid-state fermentation.
Brazilian Journal of Microbiology 39:712-717.

SAS Institute (2011) SAS/STAT User's Guide. Software Version 9.3. Cary, North Carolina, USA. 452 p.

Schuster A. and M. Schmoll (2010) Biology and biotechnology of Trichoderma. Applied Microbiology and Biotechnology 87:787799, https://doi.org/10.1007/s00253-010-2632-1

Stefanova N. M. (2007) Introducción y eficacia técnica del biocontrol de fitopatógenos con Trichoderma spp. en Cuba. Fitosanidad 11:75-79.

Vargas-Hoyos H. A. y E. Gilchrist-Ramelli (2015) Producción de enzimas hidrolíticas y actividad antagónica de Trichoderma asperellum sobre dos cepas de Fusarium aisladas de cultivos de tomate (Solanum lycopersicum). Revista Mexicana de Micología 42:916.

Veena G. A. and N. P. E. Reddy (2014) Mycoparasitism of Trichoderma spp. on Rhizoctonia bataticola, the causal agent of dry root rot of chickpea. International Journal of Applied Biology and Pharmaceutical Technology 5:195196. 
